\title{
Molecular features of giant-cell carcinoma of the lung: a case report and literature review
}

This article was published in the following Dove Press journal:

OncoTargets and Therapy

Xin Li*

Zihe Zhang*

Jinghao Liu

Dan Wang

Sen Wei

Jun Chen

Department of Lung Cancer Surgery, Tianjin Key Laboratory of Lung Cancer Metastasis and Tumor Microenvironment, Tianjin Lung Cancer Institute, Tianjin Medical University General Hospital, Tianjin, People's Republic of China

*These authors contributed equally to this work

Correspondence: Jun Chen

Department of Lung Cancer Surgery,

Tianjin Key Laboratory of Lung Cancer

Metastasis and Tumor Microenvironment,

Tianjin Lung Cancer Institute, Tianjin

Medical University General Hospital, 154

Anshan Rd, Heping Qu,Tianjin, 300052,

People's Republic of China

Email huntercj2004@qq.com

\begin{abstract}
Giant-cell carcinoma of the lung (GCCL) is a rare histological form of poorly differentiated non-small-cell lung cancer, which is classified as a subtype of pulmonary sarcomatoid carcinomas. In this case report, we describe the case of a 50-year-old Chinese male who presented with a pulmonary nodule in the right upper lobe of his lung. After thoracoscopic lobectomy, a histopathologic diagnosis of GCCL was made. He did well postoperatively, showing no local recurrence or distal disease in a 7-year follow-up period. Furthermore, for this case, we also analyzed 295 tumor-related driver genes with high-throughput sequencing technology. We found that treatment using MEK inhibitor, CDK 4/6 inhibitor, and TP53 inhibitor may provide a new therapeutic direction for GCCL. Therefore, complete tumor excision is the best choice of treatment strategy at the early stage of GCCL and gene target therapy may be a new therapeutic option for this disease.
\end{abstract}

Keywords: giant-cell carcinoma of the lung, high-throughput sequencing, gene mutation

\section{Introduction}

According to the 2004 World Health Organization (WHO) classification of lung tumors, giant-cell carcinoma of the lung (GCCL) is classified as a subtype of pulmonary sarcomatoid carcinomas (PSCs). ${ }^{1}$ PSC accounts for $2 \%-3 \%$ of all lung cancers and is associated with smoking, which is more common in males. The mean age of onset is about 59 years, and generally, no specific signs or symptoms are observed. ${ }^{2-4}$ There are five subtypes of PSCs: polymorphic carcinoma, spindle cell carcinoma, giant-cell carcinoma, carcinosarcoma, and pulmonary blastoma. ${ }^{5}$ Herein, we report the case of a patient who presented with complaints of cough and bloody sputum, and received the diagnosis of GCCL followed by tumor resection in the right lung.

\section{Case presentation}

Seven years ago, a 50-year-old Chinese male with cough and expectoration (black phlegm) for about 2 weeks, sometimes accompanied with bloody sputum and breathlessness, was admitted to our hospital. These symptoms developed without apparent cause and in the absence of problems such as chest pain and/or distress, fever, nausea, or vomiting. A computed tomography (CT) scan of his chest showed a rough-edged shadow measuring nearly $32 \times 22 \times 10 \mathrm{~mm}$ in the right upper lobe of his lung, as shown in Figure 1 (A: before surgery; B: after surgery). The patient acknowledged several chronic disorders, including a 10-year history of bronchitis and emphysema, and was a smoker with a 30-pack-year history. The patient had no family history of lung cancer. There was no obvious abnormality noted in his system review. The results of a peripheral blood count, baseline serum chemistry screening, and urinalysis were normal on 

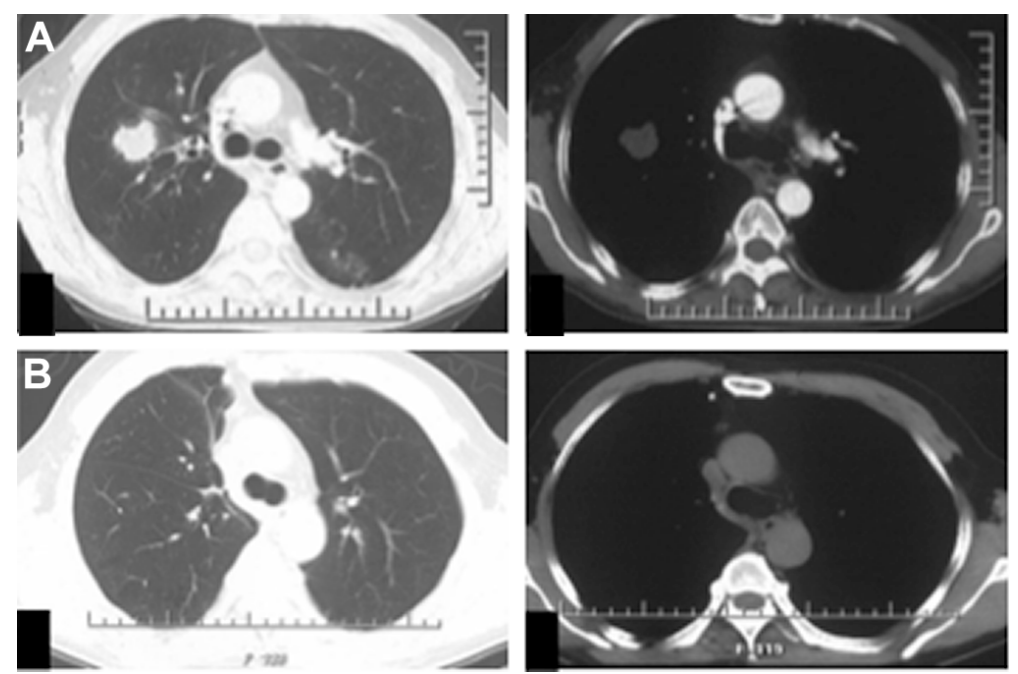

Figure I Computed tomography (CT) scans of the chest. (A) CT scan before surgery: tumor-like mass in the right upper lobe with blurred contour and irregular margin shown in the lung and mediastinal windows. (B) The 7-year follow-up CT scan after surgery.

admission, and tumor biomarker tests and a purified protein derivative test for tuberculosis were also negative. A roughedged shadow of his right upper lobe was once again seen on an enhanced CT scan of his chest, although a CT scan of his abdomen, magnetic resonance imaging of his brain, and a bone scan were all normal. His right bronchial tree also appeared normal on bronchoscopic examination, with no indication of malignancy in the biopsy and washings procured.

A right-sided upper lobectomy and systematic mediastinal lymphadenectomy were performed. A tumor of his right upper lobe, roughly $3 \mathrm{~cm}$ in diameter, was evident at surgery, without puckering of visceral pleura. Microscopic sections showed the following findings: there were many large, multicore, and bizarre giant cells, without special arrangement; cytoplasm was moderate to abundant, dense, and eosinophilic; nucleus was pleomorphic and phylloid; and the tumor cells appeared to have lost adhesion, and to be separated and embedded in a fibrous myxoid stroma. Histopathologic examination revealed the tumor cells were positively reactive to cytokeratin (CK) and vimentin, while immunostaining for chromogranin A (CgA) and synaptophysin (Syn) was negative, as shown in Figure 2. Diagnostically, vimentin is the major cytoskeletal component of mesenchymal cells and is often used as a marker of mesenchymally derived cells or cells undergoing an epithelial-to-mesenchymal transition during metastatic progression. Here, it was used as a sarcoma tumor marker to identify mesenchyme. Syn is often combined with $\mathrm{CgA}$ as a specific marker to identify tumors arising from neural and neuroendocrine tissues, such as neuroblastoma, carcinoid, and small-cell carcinoma. Therefore, the above findings supported the diagnosis of a GCCL. The detection of bronchial stump and mediastinal lymph nodes was negative,
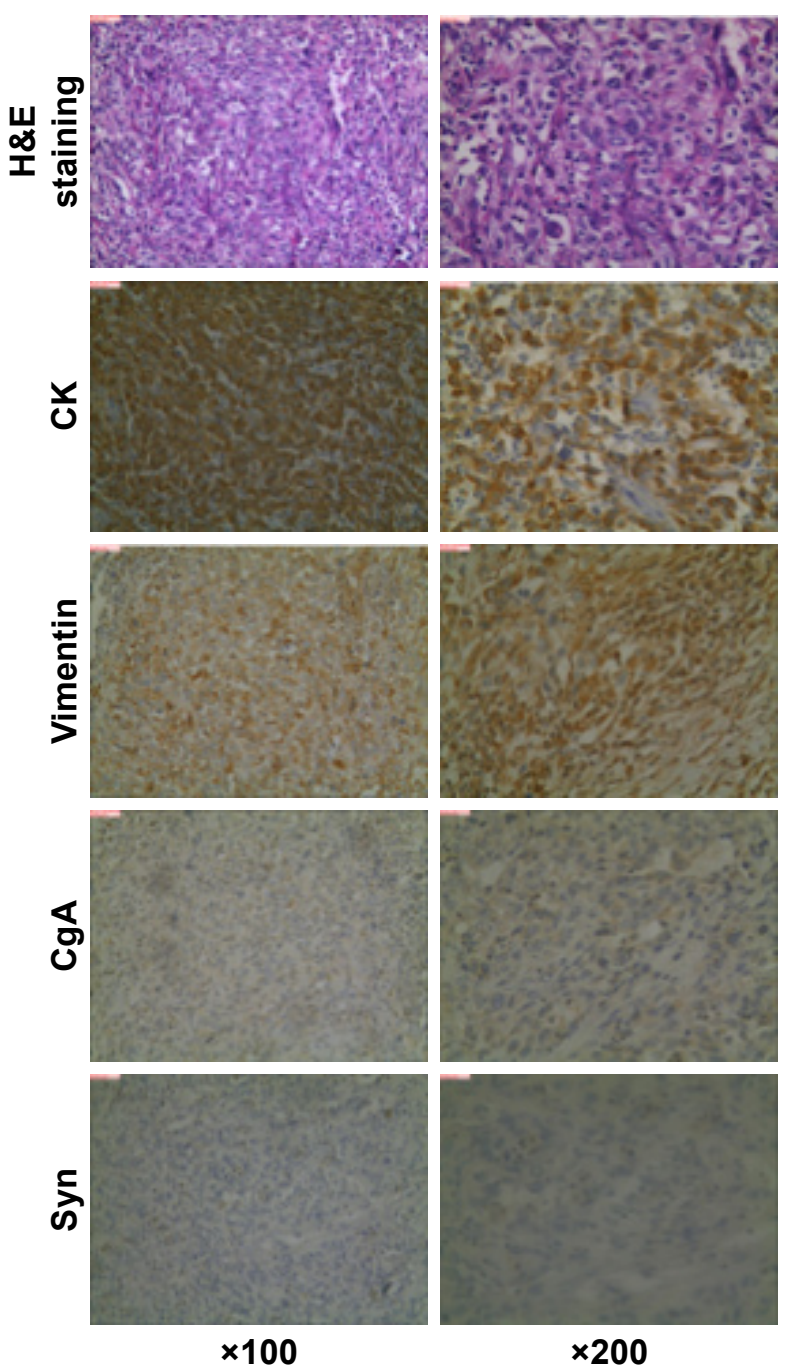

Figure 2 Pathological characteristics demonstrated by H\&E staining and immunohistochemistry. CK and vimentin immunostaining was positive, while $\mathrm{CgA}$ and Syn immunostaining was negative.

Abbreviations: $\mathrm{CgA}$, chromogranin $\mathrm{A}$; CK, cytokeratin; H\&E, hemotoxylin and eosin; Syn, synaptophysin. 
and it indicated that there was no lymph node metastasis in this patient.

Since 2 months after surgery, the patient was treated with combined chemotherapy using gemcitabine $\left(1,000 \mathrm{mg} / \mathrm{m}^{2}\right.$ on days 1 and 8 ) and cisplatin $\left(75 \mathrm{mg} / \mathrm{m}^{2}\right.$ on day 1$)$ every 3 weeks for four cycles, and then whole-body radiography examinations were carried out every year in a local hospital. He was still alive 7 years after surgery, free from disease progression. Written informed consent was obtained from the patient for the publication of this case report and the accompanying images.

\section{Follow-up and outcomes}

Until now, the therapeutic result of this case is satisfactory. With the development of high-throughput sequencing technology, more and more genomes have been successfully sequenced. Recently, in order to explore the molecular structure of this tumor, mutations of 295 tumor-related driver genes were detected by a high-throughput sequencing test (Burning Rock Biotechnology Inc., Guangzhou, People's Republic of China). All the 295 genes are listed in Table S1. We analyzed normal and tumor tissues of this patient, after ruling out genetic variations; nine tumor-related somatic mutations were confirmed, of which six were missense variants and three were synonymous variants. The highthroughput screening results of the patient are shown in Table S2. The six missense mutations included KRAS, KMT2D, TP53, SETD2, TET2, and MAP3K. As a hotspot mutation, $K R A S$ gene mutation on chromosome 12, exon 2 p.G12D, with a mutant abundance of about $26.7 \%$, indicated the tumor may be sensitive to MEK inhibitor and CDK 4/6 inhibitor like trametinib and abemaciclib. TP53 gene mutation on chromosome 17, exon 7 p.R249K, with a mutant abundance of about $8.30 \%$, indicated the tumor cell may be sensitive to some novel compound that can reverse the TP53 mutation like APR-246. For the other four somatic mutations, including KMT2D, SETD2, TET2, and MAP3K, there was no sufficient evidence to show their correlation with target drugs known so far in clinical use.

\section{Discussion and review of the literature}

GCCL is a rare malignant form of large-cell lung carcinoma, a subtype of PSCs. Nash and Stout first described this tumor in $1958 .^{6}$ For the past 25 years, the WHO has been under constant pressure to change the classification of sarcomatoid tumors of the lung, and this has been driven by our evolving understanding of their histogenetic origins. In the second revision (1981) of the WHO classification, GCCL was considered as a subtype of large-cell carcinomas. ${ }^{7}$ In the third revision (1999) of the WHO classification, it was placed within a subtype called "carcinomas with pleomorphic, sarcomatoid, or sarcomatous elements", along with pleomorphic carcinoma, spindle cell carcinoma, carcinosarcoma, and pulmonary blastoma, which are related variants. ${ }^{8}$ The rules for classifying PSC under WHO classification in 2004 were identified as more improved than in the past, for now accurate classification of a pulmonary carcinoma as a GCCL requires that the entire tumor consists only of malignant giant cells. Therefore, complete sampling of the entire tumor obtained via a surgical resection is absolutely necessary for a precise diagnosis of GCCL to be made. ${ }^{9}$

Due to the lack of accurate data from a large sample, the incidence and mortality of GCCL is generally unclear until now. It is known to be a very rare tumor variant of all races. Travis et al described in their study of a database of more than 60,000 patients in the US that GCCL comprised about $0.3 \%-0.4 \%$ of primary lung cancers, with an incidence of about three new cases per million persons per year. ${ }^{10}$ However, the GCCL incidence was much lower than other four PSC subtypes, in a series of 4,212 lung cancer cases, in which only one $(0.024 \%)$ case was confirmed to be a pure giant-cell component after complete sectioning of all tumor samples. ${ }^{11}$ Like other non-small-cell lung cancers (NSCLCs), GCCL can invade in any lung lobes, but it is observed much more frequently in the upper lobes and always at periphery. ${ }^{12}$ Usually, patients with GCCL complain of no specific signs or symptoms, but experience cough or hemoptysis, or may be asymptomatic just like other NSCLC patients. GCCL is a poorly differentiated tumor with early metastasis through lymph and blood circulation to the brain, bone, adrenal gland, and liver, similar to other NSCLCs, but uncommon metastasis to the gastrointestinal tract, kidney, and heart is reported constantly. ${ }^{2,13,14}$

GCCL is essentially undifferentiated and usually appears as a bulky necrotic mass, having no distinct architectural pattern. Under microscope, the background of GCCL contains plenty of neutrophils and lymphocytes. The tumor cells are big, multi-core, and bizarre, and the size of the tumor cells varies by more than fivefold. The tumor cells have an abundant, thick, and well-demarcated cytoplasm. The nucleus is oval or irregular and centrifugal. Multinucleated giant cells are observed more often than uninuclear cells. The size of nucleus is more than five times that of normal lymphocytes. The nuclear membrane is thin, and nuclear chromatin is coarsely granular, while the nucleolus is single and round. In cytological preparations, giant cells typically appear as single cells or in flat loose clusters, and occasionally 
in fascicles. ${ }^{15}$ Another specific feature known about this malignancy is the presence of a phenomenon called emperipolesis: collections of polymorphonuclear leukocytes within the giant cells, apparently phagocytosing cytoplasmic contents. ${ }^{6}$ However, the etiology of emperipolesis in GCCL is unclear. Some exocrine features of GCCL are also analyzed under electron microscope. The immunohistochemical features of GCCL are also nondistinct. In Rossi et al's study, CK7 was positive in two of three (67\%) GCCL samples, thyroid transcription factor-1 was negative in one of three (33\%) GCCL samples, and CK20 was negative in all three samples $(100 \%) .{ }^{16}$

GCCL does not have specific radiologic characteristics. Only Park et al described in their paper that on positron emission tomography scanning, GCCL was found to have exceedingly high standardized uptake values for radioactive glucose, values that were statistically significantly higher than other histological variants of lung cancer, and so it was difficult to diagnose with only radiologic imaging such as $\mathrm{CT}$ and magnetic resonance imaging.

For limited-stage GCCL, surgical resection is an effective treatment and provides adequate local control. ${ }^{17}$ However, most cases of GCCL are quite advanced at the time of diagnosis ( $50 \%$ of patients present with metastasis situation), ${ }^{13,18}$ and even after surgery, most patients experience tumor recurrence or death within 16-18 months. ${ }^{19}$ Platinum-based chemotherapy has been used to treat GCCL by most clinicians, but plenty of studies demonstrate the poor response of GCCL to chemotherapy in general. ${ }^{13,19-22}$ There is no study in the literature showing the efficacy of radiotherapy in the treatment of GCCL until now.

Because of the higher rate of resistance to conventional chemotherapy than other NSCLCs, we need more treatment options like molecular targeted therapy for patients with GCCL. However, molecular structure features of GCCL are still mysterious. A clinical trial involving 42 pleomorphic carcinomas was conducted to investigate the somatic mutations of EGFR and TP53. EGFR mutations were detected in $23.8 \%$ (10 of 42) of cases, including the exon 19 and 21 mutations. TP53 mutations were found in $28.6 \%$ ( 12 of 42 ) of cases. This indicated pleomorphic carcinomas are likely to benefit from treatment with EGFR tyrosine kinase inhibitors. ${ }^{23}$ Liu et al performed a next-generation sequencing (NGS) of 36 PSC patients (excluding carcinosarcoma and blastoma). They defined a broad mutation spectrum for PSC, including several known oncogenes and tumor suppressor genes (MET, KRAS, TP53, PIK3CA, STK11, NOTCH1, SMARCA4, RB1, and MLL2), and six novel mutated genes, including RASA1, CDH4, CDH7, LAMB4,
$S C A F 1$, and $L M T K 2$. From the results, they found a distinctly high frequency of splice-site somatic mutations leading to MET exon 14 skipping ( 8 of $36,22 \%$ ), mutually exclusive with known driver mutations like KRAS, EGFR, BRAF, and ALK. Further experiments indicated that ablation of METdriven signaling inhibited cell growth in H596 and Hs746T cell lines with MET exon 14 skipping. ${ }^{24} \mathrm{~A}$ few months ago, we reported the NGS result of seven pleomorphic carcinomas. A total of 136 putative somatic variants and one gene fusion were identified, of which 16 variants were considered as hotspot mutations, including EGFR, EML4-ALK, MET, BRAF, PIK3CA, and TP53. All cases were pleomorphic carcinomas, including mixed components (giant cells, spindle cells, and/or adenocarcinoma cells). EGFR mutation was found in one pleomorphic carcinoma sample with more than $10 \%$ adenocarcinoma components, which indicated that point mutation may occur in adenocarcinoma area. However, EML4-ALK gene fusion was discovered in one sample comprising entirely spindle cells and giant cells, and no KRAS gene mutation was found in any sample..$^{25}$ In this case, the tumor comprised pure giant cells, and missense mutation of the KRAS, TP53, KMT2D, SETD2, TET2, and MAP3K was detected. This result was similar to the finding of Terra et al. They reported 10 of 33 PSCs harbored KRAS mutations, and 19 cases had TP53 mutations. ${ }^{26}$ There are no relevant target drugs or clinical trials on KMT2D, SETD2, TET2, and MAP3K mutations until now. A selective allosteric inhibitor of MEK1/MEK2, trametinib, showed similar progression-free survival and response rates as docetaxel in patients with previously treated KRAS-mutation-positive NSCLC in clinical trial GSK1 120212. ${ }^{27}$ The JUNIPER study (NCT02152631) with abemaciclib, an inhibitor of CDK4 and CDK6, showed a safety profile allowing continuous dosing to achieve sustained target inhibition. The first-inhuman experience demonstrates single-agent activity for patients with advanced breast cancer, NSCLC, and other solid tumors. ${ }^{28}$ The TP53 molecule is a nuclear protein with a tumor suppressor function related to sequence-specific DNA binding and repair of damaged DNA. Generally, mutant TP53-carrying tumors reveal poorer sensitivity to conventional chemotherapy and have worse prognosis than wild-type tumors. This is a possible reason for chemotherapy resistance of GCCL. The high incidence of TP53 mutation in human tumors made it a potential target for new-concept cancer therapy. APR-246 is an effective compound which can reverse the TP53 mutation in tumor cells by synergizing with many DNA-damaging cytotoxic drugs and has little toxicity on normal cells. ${ }^{29}$ However, the effect of APR-246 on GCCL needs more experimental confirmation. 


\section{Conclusion}

GCCL is a poorly differentiated non-small-cell lung cancer. Throughout the decades, the classification and concepts pertaining to the histogenesis have been controversial before the five subtypes of PSCs were established by the WHO. GCCL has exceptionally aggressive characteristics, and its prognosis is much poorer than other NSCLCs. Although complete tumor excision and chemotherapy are often adopted in clinical use, the tumor seems to have a poor response to any currently known treatment modality, including platinum-based chemotherapy and radiotherapy. More studies are needed to deeply understand the molecular structure of GCCL, and target therapy using MEK inhibitor, CDK 4/6 inhibitor, and TP53 mutation reversal drugs may be a potent treatment option for this disease in future.

\section{Acknowledgments}

This research was partially supported by grants from the National Natural Science Foundation of China (81773207 and 61573251), the Tianjin Natural Science Foundation (16PTSYJC00160 and 16JCZDJC34200), and the Tianjin Medical University General Hospital Young Incubation Foundation (ZYYFY2015027). The funders had no role in study design, data collection and analysis, decision to publish, or in the preparation of the manuscript.

\section{Author contributions}

Xin Li and Jun Chen wrote the manuscript and analyzed all of the data. Xin Li, Zihe Zhang, Jinghao Liu, and Sen Wei provided medical care for the patients and collected the data. Dan Wang assisted in the pathological analysis. All authors contributed toward data analysis, drafting and critically revising the paper, gave final approval of the version to be published, and agree to be accountable for all aspects of the work.

\section{Disclosure}

The authors report no conflicts of interest in this work.

\section{References}

1. Teng XD. [World Health Organization classification of tumours, pathology and genetics of tumours of the lung]. Zhonghua Bing Li Xue Za Zhi. 2005;34:544-546. Chinese [with English abstract].

2. Choi HS, Seol H, Heo IY, et al. Fine-needle aspiration cytology of pleomorphic carcinomas of the lung. Korean J Pathol. 2012;46(6):576-582.

3. Paleiron N, Tromeur C, Gut-Gobert C, et al. Particularites cliniques et evolutives des carcinomes sarcomatoides du poumon: a propos d'un cas [Pulmonary sarcomatoid carcinoma: clinical and prognostic characteristics, a case report]. Rev Pneumol Clin. 2012;68:27-30. French [with English abstract].

4. Shen XY, Lin ZF, Lin Q, et al. Pulmonary sarcomatoid carcinoma: a case report. Contemp Oncol (Pozn). 2013;17:210-213.

5. Vieira T, Duruisseaux M, Ruppert AM, Cadranel J, Antoine M, Wislez M. Carcinomes sarcomatoides pulmonaires [Pulmonary sarcomatoid carcinoma]. Bull Cancer. 2012;99:995-1001. French [with English abstract].

6. Nash AD, Stout AP. Giant cell carcinoma of the lung; report of 5 cases. Cancer. 1958;11:369-376.
7. World Health Organization. Histological typing of lung tumours Tumori. 1981;67:253-272.

8. Brambilla E, Travis WD, Colby TV, Corrin B, Shimosato Y. The new World Health Organization classification of lung tumours. Eur Respir J. 2001;18:1059-1068.

9. Chen G, Yi XH. [Pathology and genetics of disease and tumours of the lung, pleura in China]. Zhonghua Bing Li Xue Za Zhi. 2005;34: 490-493. Chinese [with English abstract].

10. Travis WD, Travis LB, Devesa SS. Lung cancer. Cancer. 1995;75: 191-202.

11. Park JS, Lee Y, Han J, et al. Clinicopathologic outcomes of curative resection for sarcomatoid carcinoma of the lung. Oncology. 2011;81: 206-213.

12. Kim TH, Kim SJ, Ryu YH, et al. Pleomorphic carcinoma of lung: comparison of CT features and pathologic findings. Radiology. 2004;232:554-559.

13. Ito K, Oizumi S, Fukumoto S, et al. Clinical characteristics of pleomorphic carcinoma of the lung. Lung Cancer. 2010;68:204-210.

14. Rashid S, Rajan D, Jacob R, et al. Colonic metastases from pleomorphic carcinoma of the lung presenting as an ileocecal intussusception. ISRN Gastroenterol. 2011;2011:137139.

15. Hiroshima K, Dosaka-Akita H, Usuda K, et al. Cytological characteristics of pulmonary pleomorphic and giant cell carcinomas. Acta Cytol. 2011;55:173-179.

16. Rossi G, Cavazza A, Sturm N, et al. Pulmonary carcinomas with pleomorphic, sarcomatoid, or sarcomatous elements: a clinicopathologic and immunohistochemical study of 75 cases. Am J Surg Pathol. 2003 ; 27:311-324.

17. Zehani A, Ayadi-Kaddour A, Marghli A, et al. Carcinomes sarcomatoides du poumon: etude retrospective de 28 cas [Sarcomatoid carcinoma of the lung: retrospective study of 28 cases]. Ann Pathol. 2014;34: 124-129. French [with English abstract].

18. Alasio TM, Sun W, Yang GC. Giant cell carcinoma of the lung impact of diagnosis and review of cytological features. Diagn Cytopathol. 2007;35:555-559.

19. Hong JY, Choi MK, Uhm JE, et al. The role of palliative chemotherapy for advanced pulmonary pleomorphic carcinoma. Med Oncol. 2009;26:287-291.

20. Krishnamurthy A, Vijayalakshmi N, Majhi U. A fatal case of pure giant cell carcinoma of the lung. $J$ Cancer Res Ther. 2011;7:363-365.

21. Pelosi G, Sonzogni A, De Pas T, et al. Pulmonary sarcomatoid carcinomas: a practical overview. Int J Surg Pathol. 2010;18:103-120.

22. Bae HM, Min HS, Lee SH, et al. Palliative chemotherapy for pulmonary pleomorphic carcinoma. Lung Cancer. 2007;58:112-115.

23. Chang YL, Wu CT, Shih JY, Lee YC. EGFR and 553 status of pulmonary pleomorphic carcinoma: implications for EGFR tyrosine kinase inhibitors therapy of an aggressive lung malignancy. Ann Surg Oncol. 2011; 18:2952-2960

24. Liu X, Jia Y, Stoopler MB, et al. Next-generation sequencing of pulmonary sarcomatoid carcinoma reveals high frequency of actionable MET gene mutations. J Clin Oncol. 2015;34:794-802.

25. Li X, Wang D, Zhao Q, et al. Clinical significance and next-generation sequencing of Chinese pulmonary sarcomatoid carcinoma. Sci Rep. $2017 ; 7: 3947$

26. Terra SB, Jang JS, Bi L, et al. Molecular characterization of pulmonary sarcomatoid carcinoma: analysis of 33 cases. Mod Pathol. 2016; 29:824-831.

27. Blumenschein GR Jr, Smit EF, Planchard D, et al. A randomized phase II study of the MEK1/MEK2 inhibitor trametinib (GSK1120212) compared with docetaxel in KRAS-mutant advanced non-small-cell lung cancer (NSCLC)dagger. Ann Oncol. 2015;26:894-901.

28. Patnaik A, Rosen LS, Tolaney SM, et al. Efficacy and safety of abemaciclib, an inhibitor of CDK4 and CDK6, for patients with breast cancer, non-small cell lung cancer, and other solid tumors. Cancer Discov. 2016; 6:740-753

29. Peng X, Zhang MQ, Conserva F, et al. APR-246/PRIMA-1MET inhibits thioredoxin reductase 1 and converts the enzyme to a dedicated NADPH oxidase. Cell Death Dis. 2013;4:e881. 


\section{Supplementary materials}

Table SI Cancer-related 295-gene panel

\begin{tabular}{|c|c|c|c|c|c|c|c|c|c|}
\hline$A B L I$ & BRAF & CHEK2 & ETV4 & FGFR4 & JAK2 & PAK3 & PALB2 & RAD5ID & STAG2 \\
\hline AKTI & BRCAI & CHUK & ETV5 & FLTI & JAK3 & PAK7 & PARPI & RAD52 & STAT4 \\
\hline АKT2 & BRCA2 & $\mathrm{ClC}$ & ETV6 & FLT3 & JUN & MREIIA & PARP2 & RAD54L & STKII \\
\hline AKT3 & BRIPI & CRBN & EWSRI & FLT4 & KDM5A & $\mathrm{MSH} 2$ & PARP3 & RAFI & SUFU \\
\hline ALK & BTGI & CREBBP & $\mathrm{EZH} 2$ & FOXL2 & KDM5C & MSH6 & PARP4 & RARA & SYK \\
\hline ALOXI2B & BTK & CRKL & FAMI 23B & GATAI & KDM6A & MTOR & PAX5 & RBI & TBX3 \\
\hline $\mathrm{APC}$ & CIIORF30 & CRLF2 & FAM46C & GATA2 & KDR & MUTYH & PBRMI & REL & TET2 \\
\hline APCDDI & CI7ORF39 & CSFIR & FANCA & GATA3 & KEAPI & MYC & PDGFRA & RET & TGFBR2 \\
\hline $\mathrm{AR}$ & CARDII & CTCF & FANCC & GNAII & KIT & MYCLI & PDGFRB & RICTOR & TIPARP \\
\hline ARAF & CASP8 & CTNNAI & FANCD2 & GNAI 3 & KLHL6 & MYCN & PDKI & RNF43 & TMPRSS2 \\
\hline ARFRPI & CBFB & CTNNBI & FANCE & GNAQ & KRAS & MYD88 & PIK3C2G & RPAI & TNFAIP3 \\
\hline ARIDIA & CBL & CUL4A & FANCF & GNAS & LMOI & MYST3 & PIK3C3 & RPTOR & TNFRSFI4 \\
\hline ARID2 & CCNDI & CUL4B & FANCG & GPRI 24 & LRPIB & NBN & PIK $3 C A$ & ROSI & TOPI \\
\hline ASXLI & CCND2 & CYPI7AI & FANCl & GRIN2A & MAP2KI & NCORI & PIK3CG & RUNXI & TP53 \\
\hline ATM & CCND3 & DAXX & FANCL & GSK3B & MAP2K2 & NFI & PIK3RI & RUNXITI & TRRAP \\
\hline ATR & CCNEI & DDR2 & FANCM & HGF & MAP2K 4 & NF2 & PIK3R2 & SETD2 & $\mathrm{TSCl}$ \\
\hline ATRX & CD79A & DIS3 & FAT3 & HLA-A & MAP3KI & NFE2L2 & PMS2 & SF3BI & TSC2 \\
\hline AURKA & CD79B & DNMT3A & FBXW7 & HRAS & MAP3KI 3 & NFKBIA & PNRCI & $\mathrm{SH} 2 \mathrm{~B} 3$ & TSHR \\
\hline AURKB & CDC73 & DOTIL & FGFIO & IDHI & MCLI & NKX2-I & PPP2RIA & SMAD2 & VHL \\
\hline$A X L$ & $\mathrm{CDHI}$ & EGFR & FGFI 2 & IDH2 & MDM2 & NOTCHI & PRDMI & SMAD4 & WISP3 \\
\hline $\mathrm{BACHI}$ & CDKI2 & EP300 & FGFI3 & IGFI & MDM4 & $\mathrm{NOTCH} 2$ & PRKARIA & SMARCA4 & WTI \\
\hline BAPI & CDK4 & EPHA3 & FGFI9 & IGFIR & MEDI 2 & NOTCH3 & PRKDC & SMARCBI & XPOI \\
\hline BARDI & CDK6 & EPHA5 & FGF23 & IGF2 & MEF2B & $\mathrm{NOTCH} 4$ & PRSS8 & SMARCDI & $\mathrm{XRCC} 3$ \\
\hline $\mathrm{BCL} 2$ & CDK8 & EPHBI & FGF3 & IKBKE & MENI & NPMI & PTCHI & SMO & ZNF2I7 \\
\hline BCL2L2 & CDKNIB & ERBB2 & FGF4 & IKZFI & MET & NRAS & PTEN & SOCSI & ZNF703 \\
\hline BCL6 & CDKN2A & ERBB3 & FGF6 & IL7R & MITF & NSDI & PTPNII & SOX10 & \\
\hline BCOR & CDKN2B & ERBB4 & FGF7 & INHBA & MLHI & NTRKI & RAD50 & SOX2 & \\
\hline BCORLI & CDKN2C & ERG & FGFRI & IRF4 & MLL & NTRK2 & RAD5 I & SPEN & \\
\hline $\mathrm{BCR}$ & CEBPA & ESRI & FGFR2 & IRS2 & MLL2 & NTRK3 & RAD5IB & SPOP & \\
\hline BLM & CHEKI & ETVI & FGFR3 & JAKI & MPL & NUP93 & RAD5IC & SRC & \\
\hline
\end{tabular}

Table S2 High-throughput sequencing results of the patient

\begin{tabular}{|c|c|c|c|c|c|c|c|}
\hline chr:posi & ref $>$ alt & Type & $\begin{array}{l}\text { Gene } \\
\text { name }\end{array}$ & $\begin{array}{l}\text { Amino acid } \\
\text { change }\end{array}$ & Effect & Impact & $\begin{array}{l}\text { Mutant } \\
\text { abundance }\end{array}$ \\
\hline $12: 253982849$ & $\mathrm{C}>\mathrm{T}$ & SNP & KRAS & p.GI2D & Missense_variant & Moderate & $26.7 \%$ \\
\hline $12: 49420858$ & $\mathrm{C}>\mathrm{T}$ & SNP & KMT2D & p.R4964H & Missense_variant & Moderate & $34.0 \%$ \\
\hline $17: 7577535$ & $\mathrm{C}>\mathrm{T}$ & SNP & TP53 & p.R249K & Missense_variant & Moderate & $8.30 \%$ \\
\hline $3: 47 \mid 47512$ & $\mathrm{~T}>\mathrm{C}$ & SNP & SETD2 & p.YI605C & Missense_variant & Moderate & $16.60 \%$ \\
\hline $4: 106164796$ & $\mathrm{G}>\mathrm{A}$ & SNP & TET2 & p.EI222K & Missense_variant & Moderate & $48.50 \%$ \\
\hline $5: 56 \mid 77065$ & $\mathrm{~A}>\mathrm{T}$ & SNP & MAPЗKI & p.T779S & Missense_variant & Moderate & $41.40 \%$ \\
\hline $12: 49434520$ & $A>G$ & SNP & $K M T 2 D$ & p.L2345= & Synonymous_variant & Low & $66.10 \%$ \\
\hline 17:78882693 & $\mathrm{C}>\mathrm{T}$ & SNP & RPTOR & p.D828= & Synonymous_variant & Low & $6.40 \%$ \\
\hline $2: 209106734$ & $\mathrm{C}>\mathrm{T}$ & SNP & IDHI & p.S278= & Synonymous_variant & Low & $42.60 \%$ \\
\hline
\end{tabular}

Abbreviations: alt, gene alteration; chr, chromosome; posi, position; ref, reference genome; SNP, single-nucleotide polymorphism.

OncoTargets and Therapy

\section{Publish your work in this journal}

OncoTargets and Therapy is an international, peer-reviewed, open access journal focusing on the pathological basis of all cancers, potential targets for therapy and treatment protocols employed to improve the management of cancer patients. The journal also focuses on the impact of management programs and new therapeutic agents and protocols on patient perspectives such as quality of life, adherence and satisfaction. The manuscript management system is completely online and includes a very quick and fair peer-review system, which is all easy to use. Visit http://www.dovepress.com/testimonials.php to read real quotes from published authors. 